\title{
Monte Carlo simulation of the cosmic muon charge ratio
}

\author{
Abd Al Karim Haj Ismail ${ }^{1,2}$ \\ ${ }^{1}$ College of Humanities and Sciences, Ajman University, PO Box 346, \\ United Arab Emirates, \\ ${ }^{2}$ Nonlinear Dynamics Research Center (NDRC), Ajman University, \\ United Arab Emirates, \\ a.hajismail@ajman.ac.ae
}

\begin{abstract}
The muonic component of air showers is sensitive to the mass and energy of the primary cosmic ray and is the most abundant component of charged particles arriving at the surface, and able to penetrate deep underground. The muon charge ratio, defined as the number of positive over negatively charged muons, is a very interesting quantity for the study of hadronic interactions at high energies and the nature of cosmic ray primaries. Furthermore, Earth's atmosphere is the development medium of cosmic air showers before they arrive at the ground. Therefore, variations in the density of the atmosphere between seasons must be studied. It is also very important to account for the zenith angular dependence of atmospheric muons, in particular for showers penetrating the atmosphere at high zenith angles.

We present a study of the muon charge ratio using Monte Carlo simulations of two cosmic primaries, proton, and iron, of $100 \mathrm{TeV}$ and $1 \mathrm{PeV}$ energies, and with a zenith angle of $0^{\circ}$ to $60^{\circ}$. The dependence on the direction of extensive air showers EAS and their radial distance appears to be very pronounced. In addition, the muon density is discussed assuming the Central European Atmosphere in June and December.
\end{abstract}

Keywords: Cosmic rays; extensive air showers; five muon charge ratio; muon density.

\section{Introduction}

Primary cosmic rays enter the Earth's atmosphere and do not simply travel the atmosphere to the ground. Instead, they interact with nuclei in the atmosphere and create cascades of secondary particles, called Extensive Air Showers (Auger et al., 1939), which consist of electromagnetic, hadronic, muonic, and neutrino components. The development of these components in the atmosphere depends on the type and energy of the primary particle. More than 100 years after their discovery, the origin of high-energy cosmic rays is still under investigation. Cosmic rays with energies around the knee (a steepening in the energy spectrum at 3-4 PeV) (Horandel, 2004) are thought to have a galactic origin, and an extra-galactic origin is assumed at high energies. Recent results from the Pierre Auger Experiment (Aab et al., 2017) measured the distribution of arrival directions of ultra-high energy cosmic rays and showed that particles with energies above $8 \times 10^{18}$ $\mathrm{eV}$ have an extra-galactic origin (Aab et al., 2017). However, many open questions about their sources and propagation mechanisms are still unanswered. Therefore, accurate analyses of 
different components of the cosmic air shower can be very useful to understand the properties of these very energetic particles.

Atmospheric cosmic ray muons are produced from the decay of these charged mesons, which will also lead to the production of atmospheric neutrinos:

$$
\begin{aligned}
& \pi^{ \pm} \rightarrow \mu^{ \pm}+v_{\mu}\left(\tilde{v}_{\mu}\right), \mu^{+} \rightarrow e^{+}+v_{e}+\tilde{v}_{\mu} \\
& K^{ \pm} \rightarrow \mu^{ \pm}+v_{\mu}\left(v_{\mu}\right), \mu^{-} \rightarrow e^{-}+\tilde{v}_{e}+\tilde{v}_{\mu}
\end{aligned}
$$

The muon charge ratio is represented by the number of positive over negative charged atmospheric muons $R_{a t m}=N_{\mu^{+}} / N_{\mu^{-}}$. Up to about $200 \mathrm{GeV} / \mathrm{c}$ of momentum, a constant muon charge ratio was obtained from measurements of various experiments, and then the ratio increases at higher momenta (Ashley et al., 1975 \& Adamson et al., 2007). Measurements of the charge ratio can provide relevant information for both cosmic ray properties and particle physics studies and can be used to constrain hadronic interaction models, study the influence of the geomagnetic field on the primary and secondary component of cosmic rays, and predict better the atmospheric neutrino flux. The magnitude and the energy dependence of the muon charge ratio are determined by the production and interaction cross-sections of mesons and by their decays. As most cosmic rays and the nuclei with which they interact have a positive charge, positive meson production is favored, therefore more positive muons are expected and $R>1$.

The muonic component is the largest component of the cascade of air showers at ground level and is very sensitive to the mass and energy of the primary cosmic particle, which is considered as the most important quantities to describe the properties of the cosmic ray primary. During the summer season, the sun heats the atmosphere while it cools down in fall, which causes changes in the temperature and density of the atmosphere. Thus, as the cosmic ray air showers develop in the atmosphere, the production and propagation of muons are highly affected by the influence of seasonal variations.

The purpose of this letter is to study the radial and azimuthal asymmetries in the lateral distribution of charged cosmic particles and to investigate the effects of the summer-winter variation in the Earth's atmosphere on the muonic component, energy, and radial dependence at sea level.

\section{Simulation}

We use the Monte Carlo air simulation software CORSIKA (Cosmic Ray Simulation for KASCADE) (Heck et al., 1998) for the simulation of cosmic air showers. Different theoretical frameworks are assumed, and CORSIKA allows us to choose between hadronic interaction models at low and high energies. SIBYLL 2.3c (Fedynitch et al., 2019) is used to describe the hadronic interaction at high energy $(\mathrm{E}>80 \mathrm{GeV})$, FLUKA (Ferrari et al., 2005) for lower energies, and EGS4 (Nelson et al., 1985) for the electromagnetic processes. Proton and ironinduced EAS are considered as the two extreme cases for the primary mass assumption. We simulate 500 air showers with energies of $10 \mathrm{TeV}, 100 \mathrm{TeV}$, and $1 \mathrm{PeV}$ according to E-1 spectrum, the incident with a zenith angle between $\left(0^{\circ}<\theta<60^{\circ}\right)$ and over a $360^{\circ}$ azimuth angle. Induced air showers of energies of $10 \mathrm{TeV}$ were only included in the study of muon density (Sec. $3.2)$. The simulation is produced 
first assuming the Central European Atmosphere for June 13, 1993, called later as "summer atmosphere" and repeated assuming the Central European Atmosphere for December 24, 1993, "winter atmosphere". Both atmospheres are configured in the CORSIKA simulation software (Heck et al., 1998).

To include the zenith dependence, the simulation is divided into two zenith bands, equivalent to two equal solid angles $\Omega=2 \pi\left(\cos \theta_{\min }-\cos \theta_{\max }\right)=1.57 \mathrm{sr}$. The first solid angle $\Omega 1 ; 1>\cos \theta>0.75$, corresponds to a zenith angle $\left[0^{\circ}-41.4^{\circ}\right]$, and air showers are called "vertical showers". The second solid angle $\Omega 2 ; 0.75>\cos \theta>0.5$, corresponds to a zenith angle $\left[41.4^{\circ}-60^{\circ}\right]$, and air showers are called "inclined showers".

\section{Results and discussion}

\subsection{Muon ratio - directional and radial dependence}

The asymmetry in the azimuthal distribution of charged air showers is mainly caused by the effect of the Earth's magnetic field and the shower attenuation in the atmosphere. An East-West asymmetry is observed due to the effect of the Earth's magnetic field on the flux of cosmic rays (Diep et al., 20014). To study this effect, the muon ratio is studied for the proton and ironinduced primaries. The azimuthal variation of the muon charge ratio for proton and iron-induced vertical and inclined showers, observed at a radial distance of $500 \mathrm{~m}$. For more inclined showers, the amount of atmosphere that showers need to traverse before arriving at the surface is larger than for more vertical showers. Therefore, inclined showers will be more attenuated in the atmosphere and this results in a more pronounced variation in the muon charge ratio (Figure 1).
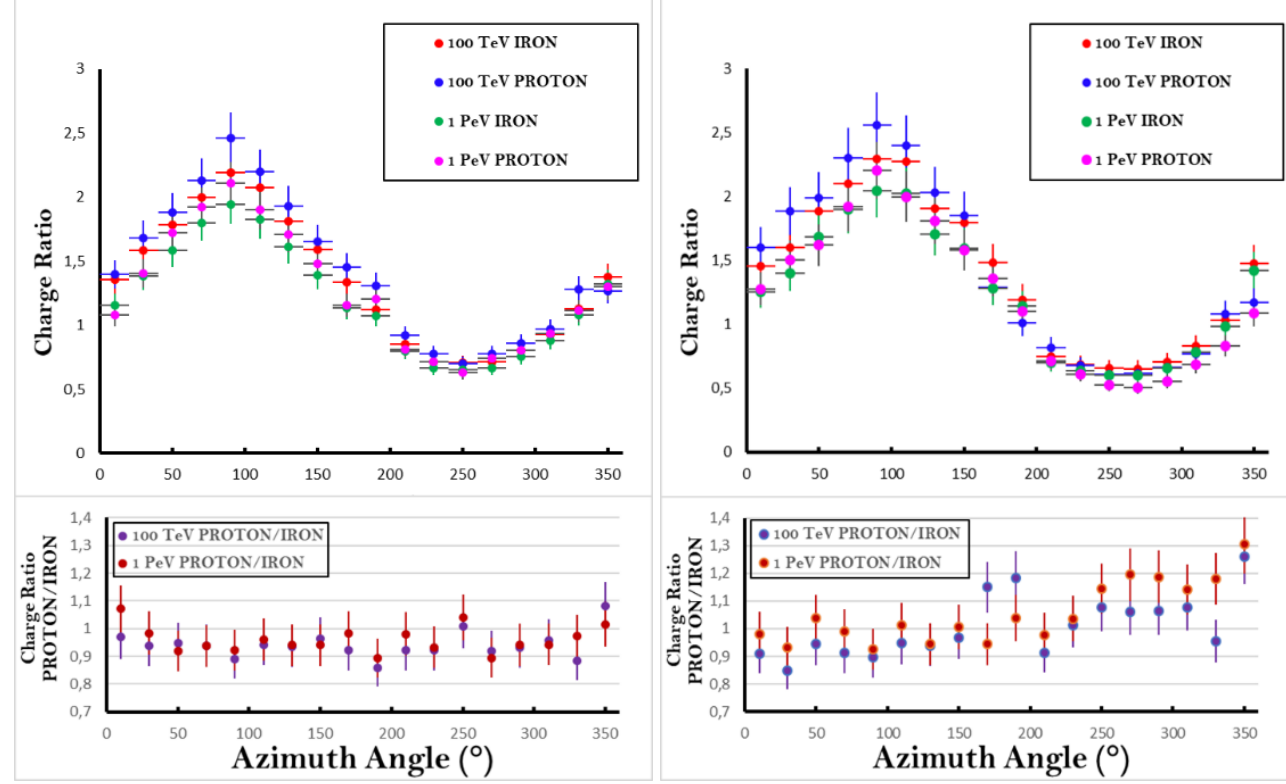

Fig. 1. Muon charge ratio as a function of the azimuth distribution for proton and iron-induced vertical (Left) and inclined air showers (Right), observed at $0-500 \mathrm{~m}$ from the shower core, and with energies of $100 \mathrm{TeV}$ and $1 \mathrm{PeV}$. 
The variation of the muon charge ratio depends also on the type and energy of the primary cosmic ray used in the simulation. This is related to the different interaction points in the atmosphere for proton and iron-induced showers and consequently leads to a different stage of development and number of muons at the surface. Therefore, the muon ratio shows a clear dependence on the radial distance to the shower core (Figure 2).
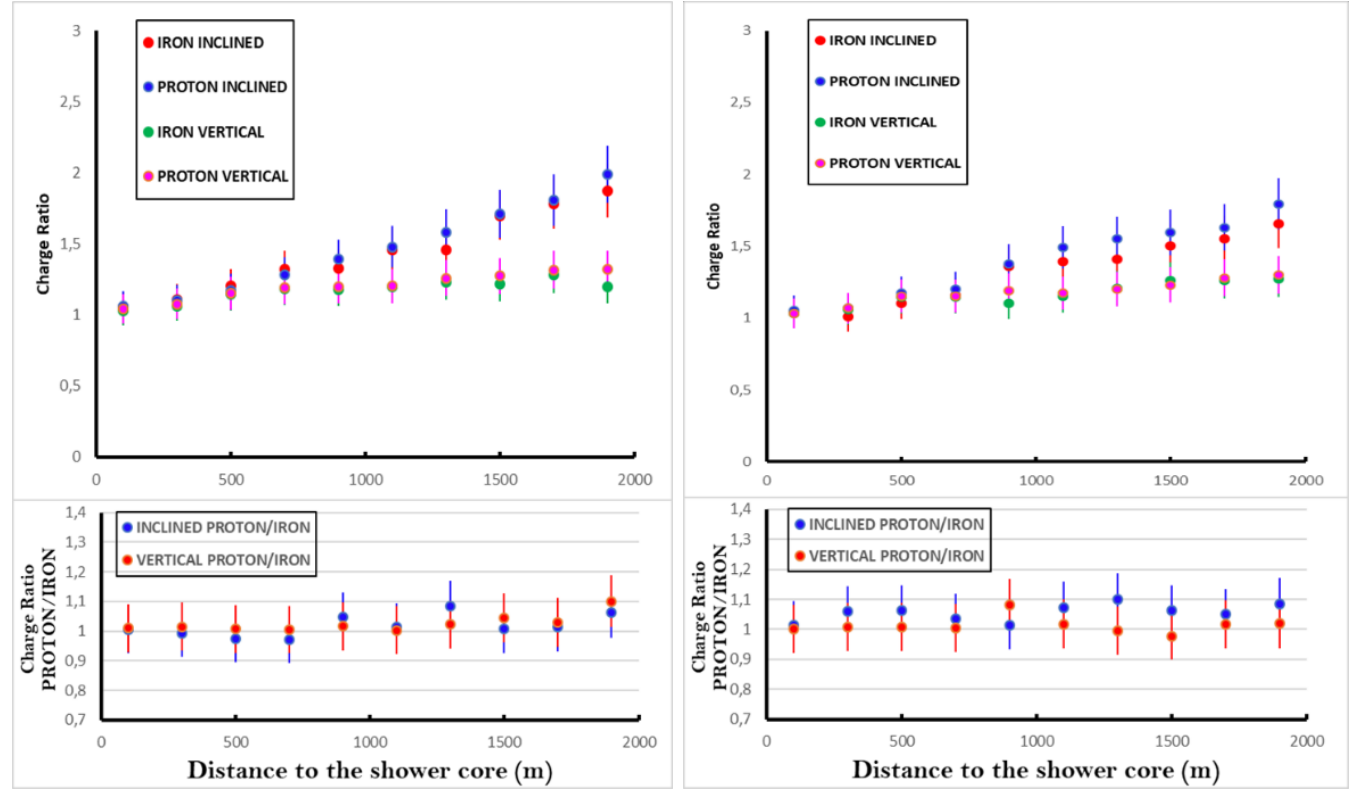

Fig. 2. Muon charge ratio as a function of the distance to the shower core for proton and ironinduced air showers, observed at vertical and inclined air showers and with energies of $100 \mathrm{TeV}$ (Left) and $1 \mathrm{PeV}$ (Right).

\subsection{Muon density}

Many cosmic ray experiments showed a pronounced variation in the muon density of cosmic air showers with increasing distances from the shower core (Bertaina et al., 2014). The relative muon density of iron and proton-induced vertical and inclined showers of different energies are presented in both summer (Figure 3) and winter (Figure 4) atmospheres. Due to the larger number of muons produced from iron showers compared to the number of muons from proton showers, the muon density is different for different primaries and distances from the shower core. The difference in the relative muon density between iron and proton-induced air showers is at its maximum close to the shower core and decreases at larger distances. In addition, a clear dependence on the primary energy is observed. Close to the shower core, the difference between the summer and winter atmosphere is smaller because of the larger number of muons produced at high energies, which results in small attenuation in the atmosphere.

The amount of atmosphere that showers need to traverse is larger for inclined than vertical showers. Therefore, inclined showers are more attenuated in the atmosphere. Consequently, the variation in the relative muon ratio is larger for inclined showers. However, this difference is also less pronounced at large distances from the shower core. 
Furthermore, particles lose their energy by interacting with air molecules in the atmosphere. Because the density of the atmosphere is larger in winter than in summer, more muons can reach the detection level in summer, the attenuation and variation of relative muon density become more notable than in the winter atmosphere.
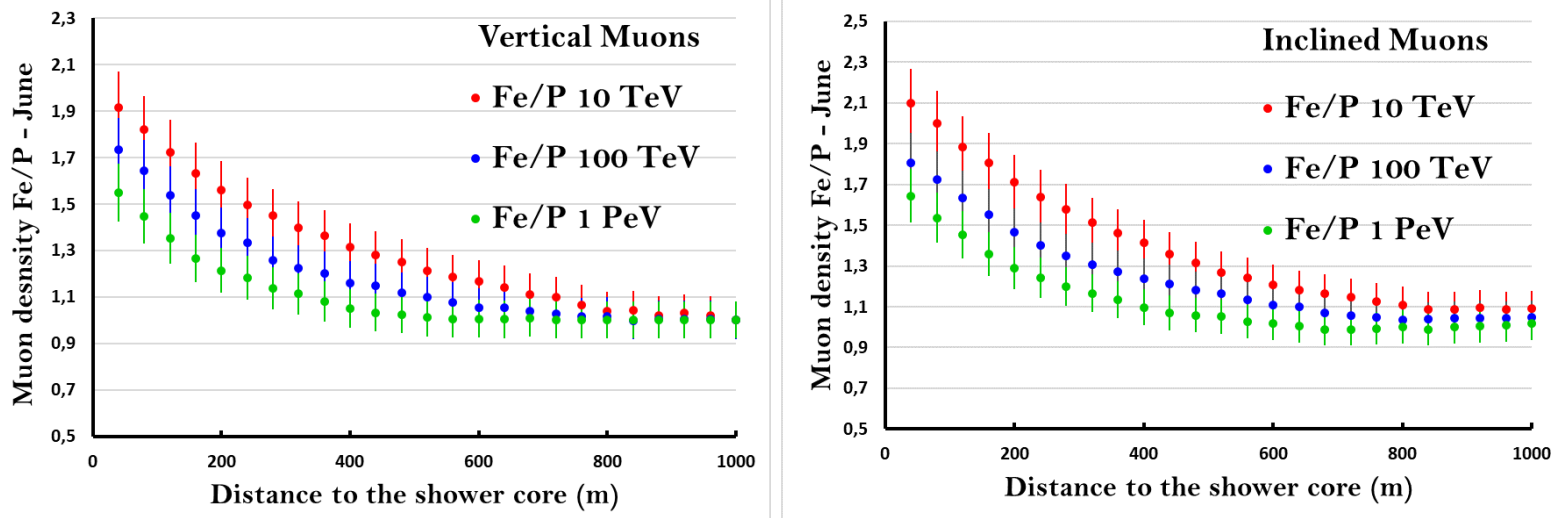

Fig. 3. The ratio of the muon density of iron and proton-induced air showers as a function of the distance to the shower core at three energy bins. Results are shown using summer Central European Atmosphere for vertical and inclined induced air showers (Left and Right, respectively).

To investigate the influence of the atmosphere on shower development, the relative muon ratio of iron and proton-induced air showers is compared between summer and winter atmospheres (Figure 5), for both vertical (Right) and inclined (Left) induced air showers, as a function of the distance to the shower core. The difference between summer and winter atmospheres is about 2-3 $\%$ for vertical showers, and increases to 3-5\% for inclined showers, with a clear energy dependence mainly for inclined induced air showers. A more detailed correction factor can be investigated when comparing to real data, like a correction for the muon multiplicity variations (Tilav el at., 2020).
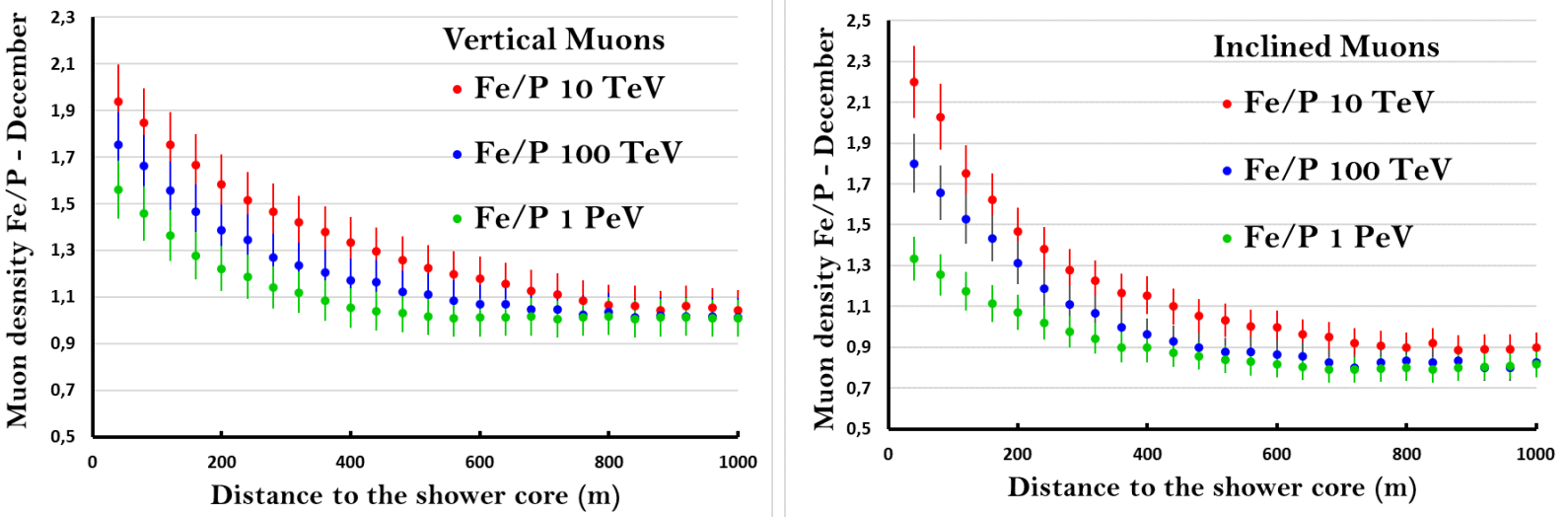

Fig. 4. The ratio of the muon density of iron and proton-induced air showers as a function of the distance to the shower core at three energy bins. Results are shown using winter Central European Atmosphere for vertical and inclined induced air showers (Left and Right, respectively). 

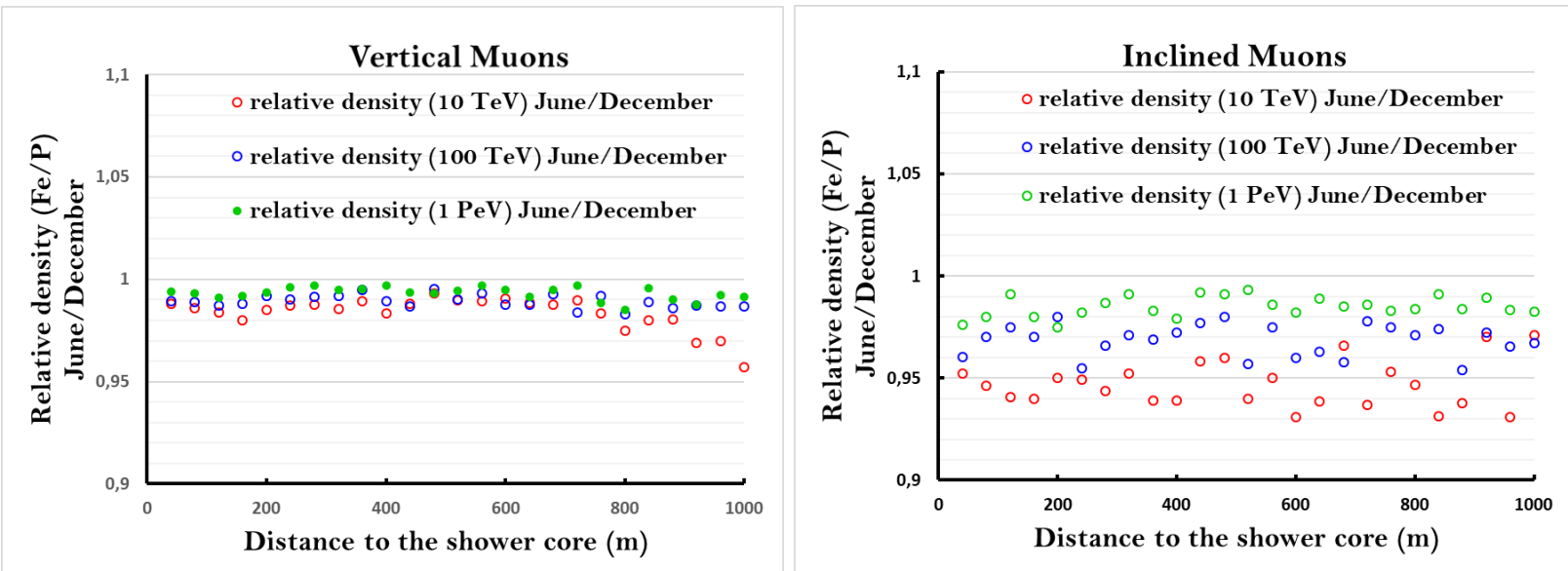

Fig. 5. The ratio of relative iron/proton muon density of summer and winter Central European Atmospheres as a function of the distance to the shower core. Results are shown for vertical (Top) and inclined (Bottom) induce air showers, and in three energy bins $(10 \mathrm{TeV}, 100 \mathrm{TeV}$, and $1 \mathrm{PeV})$.

\section{Conclusion}

We have investigated the muon charge ratio of cosmic air showers using Monte Carlo simulations of proton and iron-induced air showers of $100 \mathrm{TeV}$ and $1 \mathrm{PeV}$ primary energy. A variation in the muon ratio is observed under the influence of the direction of the air shower and the distance from the shower core at the detection level. Furthermore, the muon density is discussed for vertical and inclined showers in two different Central European Atmospheres (June and December). In conclusion, results of the relative muon density of iron and proton showers showed a significant radial, directional, and energy dependence.

Besides giving insight into the dependence of the muon charge ratio on the azimuthal distribution, the method of splitting the simulation into two sets of showers, based on their incoming direction, enabled us to discuss the variation in the muon charge ratio of vertical and inclined air showers. Applying the results to real data, such as IceCube data, could be useful to check the seasonal variation of atmospheric muons.

The need for systematic simulations of high-energy showers and in a wide energy range is very time-consuming. Such a task is of great help for analyses of larger zenith angles. In addition, this work used SIBYLL 2.3, FLUKA, and EGS4 in the simulation, however, there exist other models that can be considered such as QGSJET (Ostapchenko, 2006) and DPMJET (Roesler et al., 2001), which can be also helpful to check the dependence on hadronic interaction models.

\section{ACKNOWLEDGEMENTS}

The author acknowledges Ajman University for supporting the research, Internal Research Grant No: [DGSR Ref. 2021-IRG-HBS-12]. 


\section{References}

Aab, A., et al. (2015). The Pierre Auger Cosmic Ray Observatory. Nuc. Inst. Meth in Physics Research Section A: Accelerators, Spectrometers, Detectors and Associated Equipment, 798:172213.

Aab, A., et al. (2017). Observation of a large-scale anisotropy in the arrival directions of cosmic rays above $8 \times 10^{18} \mathrm{eV}$, Science, 357:1266-1270.

Adamson, P., et al. (2007). Measurement of the atmospheric muon charge ratio at TeV energies with the MINOS detector, Physical Review D, 76(5):052003.

Ashley, G.K., Keuffel, J.W. \& Larson, M.O. (1975) . Charge ratio of ultra-high-energy cosmicray muons, Physical Review D, 12(1):20-35.

Auger, P., Ehrenfest, P., Maze, R., Daudin, R. \& Freon, R.A. (1939). Extensive cosmic-ray showers. Reviews of Modern Physics, 11: 288-291.

Bertaina, M. (2014). The cosmic ray spectrum and composition measured by KASCADE-Grande between $10^{16} \mathrm{eV}$ and $10^{18} \mathrm{eV}$, Nuclear Physics B - Proceedings Supplements, 256-257, 149-160.

Diep, P.N., Dinh, P.N., Duong, N.H., Nhung, P.T.T., Darriulat, P., Thao, N.T., Thieu, D.Q., \& Thuan, V.V. (2004). Measurement of the east-west asymmetry of the cosmic muon flux in Hanoi, Nuclear Physics B, 678:3-15.

Fedynitch, A., Riehn, F., Dembinski, H.P., Engel, R., Fedynitch, A., Gaisser, T.K. \& Stanev, T. (2019). The hadronic interaction model sibyl 2.3c and Feynman scaling, Physical Review D, 100:103018.

Ferrari, A., Sala, P.R., Fasso, A. \& Ranft, J. (2005). FLUKA: A multi-particle transport code (Program version 2005), CERN-2005-010, SLAC-R-773, INFN-TC-05-11, CERN-2005-10.

Heck, D., Knapp, J., Capdevielle, J. N., Schatz, G. \& Thouw, T. (1998). Corsika: A monte carlo code to simulate extensive air showers.

Horandel, J.R. (2004). Models of the knee in the energy spectrum of cosmic rays, Astroparticle Physics, 21:241-265.

Nelson, W.R., Hirayama, H. \& Roger, D.W.O. (1985). EGS4 code system, SLAC Report No. SLAC-265.

Ostapchenko, S. (2006). QGSJET-II: towards the reliable description of very high energy hadronic interactions, Nuclear Physics B - Proceedings Supplements, 151:143-146.

Roesler, S. and Engel, R. \& Ranft, J. (2001). DPMJET-III, a hadronic interaction model for cascade simulations, Proceedings of the 27th International Cosmic Ray Conference. Hamburg, Germany, 2:439. 
Tilav, S., Gaisser, T.K., Soldin, D., \& Desiati, P. (2020). Seasonal variation of atmospheric muons in IceCube, Pos, ICRC2019: 894.

Submitted: $23 / 12 / 2020$

Revised: $\quad 19 / 01 / 2021$

Accepted: 06/02/2021

DOI: $10.48129 /$ kjs.v49i1.11497 